\title{
Sex- and age-specific normative values for handgrip strength and components of the Senior Fitness Test in community-dwelling older adults aged $65-75$ years in Germany: results from the OUTDOOR ACTIVE study
}

\author{
Birte Marie Albrecht ${ }^{*}$ (D), Imke Stalling and Karin Bammann
}

\begin{abstract}
Background: Physical fitness is a key component of independent living and healthy ageing. For the measurement of physical fitness in older adults, the Senior Fitness Test is a commonly used tool. The objective of this study is to calculate sex- and age-specific normative values for handgrip strength and components of the Senior Fitness Test for older adults (65-75 years) in Germany.

Methods: Cross-sectional data of 1657 community-dwelling older adults residing in Bremen, Germany (53\% female) were included in this study. Physical fitness was assessed using the following measurements of the Senior Fitness Test battery: 30s-chair stand test, 2 min-step test, sit-and-reach test, and back scratch test. In addition, handgrip strength was measured using a Saehan DHD-3 digital hand dynamometer SH1003. Sex- and age specific normative values were calculated for the 1st, 3rd, 10th, 25th, 50th, 75th, 90th, 97th, and 99th percentile using the GAMLSS method.

Results: The normative values show differences dependent on sex and age. For handgrip strength, the 30s-chair stand test and the 2 min-step test, normative values were higher for men, while women reached higher values in the sit-and-reach test and the back scratch test. For both, men and women, normative values declined with age.

Conclusions: This study provides sex- and age-specific normative values for handgrip strength and components of the Senior Fitness Test for older adults in Germany. They might be useful for future research and for the application in practice.
\end{abstract}

Keywords: Elderly, Functional fitness, GAMLSS, Germany, Handgrip strength, Normative values, Older adults, Physical fitness, Reference values, Senior fitness test

\footnotetext{
* Correspondence: b.albrecht@uni-bremen.de

Institute for Public Health and Nursing Sciences (IPP), University of Bremen, Grazer Straße 2a, 28359 Bremen, Germany
}

C C The Author(s). 2021 Open Access This article is licensed under a Creative Commons Attribution 4.0 International License, which permits use, sharing, adaptation, distribution and reproduction in any medium or format, as long as you give appropriate credit to the original author(s) and the source, provide a link to the Creative Commons licence, and indicate if changes were made. The images or other third party material in this article are included in the article's Creative Commons licence, unless indicated otherwise in a credit line to the material. If material is not included in the article's Creative Commons licence and your intended use is not permitted by statutory regulation or exceeds the permitted use, you will need to obtain permission directly from the copyright holder. To view a copy of this licence, visit http://creativecommons.org/licenses/by/4.0/ The Creative Commons Public Domain Dedication waiver (http://creativecommons.org/publicdomain/zero/1.0/) applies to the data made available in this article, unless otherwise stated in a credit line to the data. 


\section{Background}

Maintaining a high level of physical fitness is required for independent living in the process of ageing $[1,2]$. Muscle strength, aerobic endurance, flexibility, and balance are needed for many activities of daily living for example running errands, carrying groceries, getting dressed, or cleaning. In addition, physical fitness is positively associated with health-related quality of life $[3,4]$ and well-being [5]. Yet, physical fitness declines with increasing age $[6,7]$.

The assessment of physical fitness is important in research as well as in practice. One of the standard tools for adults aged 60 years and above is the Senior Fitness Test which was introduced by Rikli \& Jones in 1999 [8]. The original version of the Senior Fitness Test includes the 30s-chair stand and arm curl test for measuring lower and upper body strength, the sit-and-reach and back scratch test for measuring lower and upper body flexibility, the 6 min-walk or the 2 min-step test for measuring aerobic endurance, and the $8 \mathrm{ft}$. up-and-go test for measuring dynamic balance [8]. The test results can be interpreted using normative values, e.g. the ones provided by Rikli \& Jones for older adults in the USA in 1999 [9]. Since then, other authors published further normative values for older adults in different parts of the world - for example Taiwan [10], Spain [11, 12], Portugal [13], Hong Kong [14], Chile [15], and Poland [16]. To the best of our knowledge, there are no normative values for the Senior Fitness Test for older adults in Germany.

The objective of this study is to provide sex- and agespecific normative values for handgrip strength and components of the Senior Fitness Test in communitydwelling adults aged 65-75 years in Germany.

\section{Methods}

\section{Study design and population}

The OUTDOOR ACTIVE study is part of the prevention research network AEQUIPA that investigates the role of physical activity as a key determinant of healthy ageing [17]. In this context, the aim of OUTDOOR ACTIVE is to develop and implement a community-based outdoor physical activity promotion program in older adults using a participatory approach. The OUTDOOR ACTIVE study consists of two parts: the pilot study (02/ 15-01/18) and the cluster-randomized trial (02/18-01/ 21). The eligibility criteria for both parts were 1 ) being between 65 and 75 years of age, 2) being noninstitutionalized, and 3) living in specific subdistricts in Bremen, Germany (pilot study: Arbergen, Hastedt, Hemelingen, Mahndorf, Sebaldsbrück; clusterrandomized trial: Blumenthal, Burg-Grambke, Gete, Lehe, Lehesterdeich, Neustadt, Ohlenhof, Ostertor). Address data were obtained from the registry office of
Bremen and eligible individuals were initially contacted via letter.

The data for the calculation of normative values were obtained during the baseline assessments of the pilot study and the cluster-randomized trial [18]. The participation in the baseline assessment included 1) a questionnaire on intrapersonal, interpersonal, and environmental determinants of physical activity, 2) a health examination including a short physical examination and a fitness test, and 3) a seven-day accelerometer measurement. The follow-up assessment took place 1 year after baseline, yet the data were not included in the following analyses.

In total, 11,079 individuals meeting the age criteria were registered in the study regions of the pilot study and the cluster-randomized trial. Of those, 461 could not participate due to acute health problems and 125 deceased. Four hundred fifty individuals moved outside the study region and 77 could not participate because of language barriers. Of the remaining 9966 confirmed eligible individuals, 3425 were never reached and 4247 refused to participate. Furthermore, 151 individuals of the subdistrict Lehesterdeich were never contacted because the end of the Lehesterdeich survey period was reached and the actual sample size of the subdistrict already exceeded the calculated sample size. Two thousand one hundred forty-three individuals participated in at least one part of the pilot study or the cluster-randomized trial and, of those, 1657 participants completed at least one physical fitness measurement and were, therefore, included in the calculation of normative values.

All participants provided written informed consent. Both the pilot study and the cluster-randomized trial were approved by the ethical committee of the University of Bremen.

\section{Measures}

The health examinations to collect data on physical fitness, anthropometry, and age took place in survey rooms in the respective subdistricts between 10/15-08/16 (pilot study) and 06/18-07/19 (cluster-randomized trial). The examinations were conducted by trained survey personnel.

The implementation of the Senior Fitness Test by Rikli \& Jones [8] to assess the physical fitness of the OUTDOOR ACTIVE participants is depicted in Table 1. Handgrip strength - measured with a Saehan DHD-3 digital hand dynamometer SH1003 (Saehan Corporation, Changwon, South Korea) - was used in lieu of the 30sarm curl test to assess upper body strength. The measurement was conducted in a standing position, upper arm close to the upper body, and elbow flexed in a $90^{\circ}$ angle. Maximum isometric strength was measured twice for both hands and the overall maximum was used for the calculation of normative values [21]. For the 30s- 
Table 1 Implementation of the Senior Fitness Test [19] in the OUTDOOR ACTIVE study

\begin{tabular}{lll}
\hline Physical fitness domain & Senior Fitness Test & OUTDOOR ACTIVE \\
\hline Upper body strength & 30s-arm curl test & Handgrip strength \\
Lower body strength & 30s-chair stand test & 30s-chair stand test \\
Aerobic endurance & 6 min-walk test & 2 min-step test \\
& OR & \\
& 2 min-step test & \\
Lower body flexibility & Sit-and-reach test & Sit-and-reach test \\
Upper body flexibility & Back scratch test & Back scratch test \\
Dynamic balance & $8 \mathrm{ft}$ up-and-go test & a \\
\hline
\end{tabular}

${ }^{a}$ Instead of dynamic balance, static balance was assessed using the 4-stage balance test [20]

chair stand test, the participant has to stand up from a seated position and sit back down as often as possible in 30 s. The 2 min-step test requires the participant to step in place for 2 min with both knees reaching a required height. During the sit-and-reach test, the participant sits on a chair with one leg extended and has to reach toward his toes. For the back scratch test, the participant has to try to touch both hands behind the back with one hand reaching over the shoulder and the other up the middle of the back. The tests were conducted according to the test protocols in the Senior Fitness Test Manual, 2nd edition [19]. As depicted in Table 1, the 4-stage balance test [20] to assess static balance was used in lieu of the $8 \mathrm{ft}$. up-and-go test as a measure of dynamic balance. For the 4-stage balance test, the participant has to hold four different positions with increasing level of difficulty for 10s. As the result variable is binary (yes/ no for each position), we could not calculate any normative values.

Height was measured with a Seca 217 mobile stadiometer (Seca GmbH \& Co. KG, Hamburg, Germany), body weight with a Kern MPC 250K100M personal floor scale (Kern \& Sohn GmbH, Ballingen, Germany), and waist circumference with a Seca 201 measuring tape (Seca GmbH \& Co. KG, Hamburg, Germany). Body mass index was calculated as the quotient of body weight (in $\mathrm{kg}$ ) and squared height (in $\mathrm{m}$ ) and, afterwards, classified into underweight, normal weight, overweight, and obesity according to the World Health Organization [22].

Sociodemographic information (sex, educational status) and self-rated health were assessed through a self-administered questionnaire. Educational status was categorized according to the International Standard Classification of Education 1997 [23]. Self-rated health was assessed with a single item from the SF-36 questionnaire [24].

\section{Statistical analyses}

For the description of the study population, sexstratified absolute and relative frequencies of the educational status, self-rated health, and body mass index were determined. Means and standard deviations were calculated for age, body weight, body height, waist circumference, and the physical fitness measurements. Descriptive analyses were performed with $\mathrm{SPSS}^{\circ}$ Statistics version 20.0 (IBM Corp., Armonk, NY, USA).

Generalized additive models for location, scale, and shape (GAMLSS) were used to estimate sex-stratified percentile curves dependent on age for all physical fitness measurements. GAMLSS are an extension of the LMS method [25] and allow not only to model for the mean, variability, and skewness of the response variable but also for kurtosis [26]. For each model, the distribution of the response variable was selected based on Akaike's information criterion (see Additional file 1). Cubic splines were used for smoothing. Sex- and agespecific percentile curves were plotted for percentiles 1 , $3,10,25,50,75,90,97$, and 99. The GAMLSS models were calculated and the results plotted using the gamlss package version 5.1-7 [27] in RStudio version 3.6.2 (RStudio, Inc., Boston, MA, USA).

\section{Results}

In total, 880 women (53.1\%) and 777 men (46.9\%) were included in the calculation of normative values. The mean age of women was $69.6 \pm 2.9$ years and of men $69.5 \pm 2.8$ years (for number of participants by sex and age see Additional file 2). The majority of women had normal weight $(41.6 \%)$ in comparison to men, where the majority was overweight (50.7\%). Most women (58.7\%) and men (56.4\%) declared their health status as good. Educational status was higher in men (advanced education: 64.1\%) compared to women (advanced education: 37.3\%) (see Table 2). Means, standard deviations, and standard errors by sex and age for all physical fitness measurements are depicted in Additional file 3.

Age- and sex-specific normative values for the physical fitness measurements are displayed in Figs. 1, 2, 3, 4, 5 (for Tables see Additional file 4). Test results differed between women and men across all physical fitness measurements with women reaching higher values in the flexibility domains (sit-and-reach test and back scratch test) and men reaching higher values in the strength and endurance domains (handgrip strength, 30s-chair stand test, and 2 min-step test). Apart from minor exceptions, test results declined with increasing age for both women and men.

\section{Discussion}

This paper provides sex- and age-specific normative values for handgrip strength and components of the Senior Fitness Test. The test results of all physical fitness measurements differed between woman and men, yet the age-specific decline was similar for both. 
Table 2 Characteristics of the study population

\begin{tabular}{|c|c|c|}
\hline Participants' characteristics & Women $(n=880)$ & Men $(n=777)$ \\
\hline & n (\%) & n (\%) \\
\hline \multicolumn{3}{|l|}{ Education } \\
\hline Basic education (ISCED level $1+2$ ) & $151(17.8)$ & $30(4.1)$ \\
\hline Specialized education (ISCED level $3+4$ ) & $381(44.9)$ & $235(31.8)$ \\
\hline Advanced education (ISCED level $\geq 5$ ) & $316(37.3)$ & $474(64.1)$ \\
\hline \multicolumn{3}{|l|}{ Body-mass-index $\left(\mathrm{kg} / \mathrm{m}^{2}\right)$} \\
\hline Underweight $(<18.5)$ & $9(1.0)$ & 0 \\
\hline Normal weight $(18.5-<25)$ & $364(41.6)$ & $211(27.2)$ \\
\hline Overweight $(25-<30)$ & $321(36.7)$ & $394(50.7)$ \\
\hline Obesity $(\geq 30)$ & $181(20.7)$ & $172(22.1)$ \\
\hline \multicolumn{3}{|l|}{ Self-rated health } \\
\hline Bad & $14(1.7)$ & $10(1.4)$ \\
\hline Less good & $126(15.0)$ & $86(11.6)$ \\
\hline Good & $492(58.7)$ & $417(56.4)$ \\
\hline Very good & $175(20.9)$ & $197(26.6)$ \\
\hline \multirow[t]{2}{*}{ Excellent } & $31(3.7)$ & $30(4.1)$ \\
\hline & Mean (SD) & Mean (SD) \\
\hline Age (years) & $69.6(2.9)$ & $69.5(2.8)$ \\
\hline Body weight $(\mathrm{kg})$ & $70.4(12.8)$ & $85.8(13.5)$ \\
\hline Body height (cm) & $162.8(6.6)$ & $176.5(6.8)$ \\
\hline Waist circumference (cm) & $88.2(12.3)$ & $100.1(11.8)$ \\
\hline Handgrip strength (kg) & $25.2(5.1)$ & $42.0(7.8)$ \\
\hline 30s-chair stand test ( $n$ in 30s) & $12.9(3.0)$ & $13.4(3.0)$ \\
\hline 2 min-step test ( $\mathrm{n}$ in 2 min) & $84.7(17.7)$ & $87.1(17.7)$ \\
\hline Sit-and-reach test $(\mathrm{cm})$ & $3.6(9.9)$ & $-3.9(11.3)$ \\
\hline Back scratch test $(\mathrm{cm})$ & $-4.5(9.0)$ & $-11.6(12.4)$ \\
\hline
\end{tabular}

ISCED International Standard Classification of Education

$S D$ standard deviation

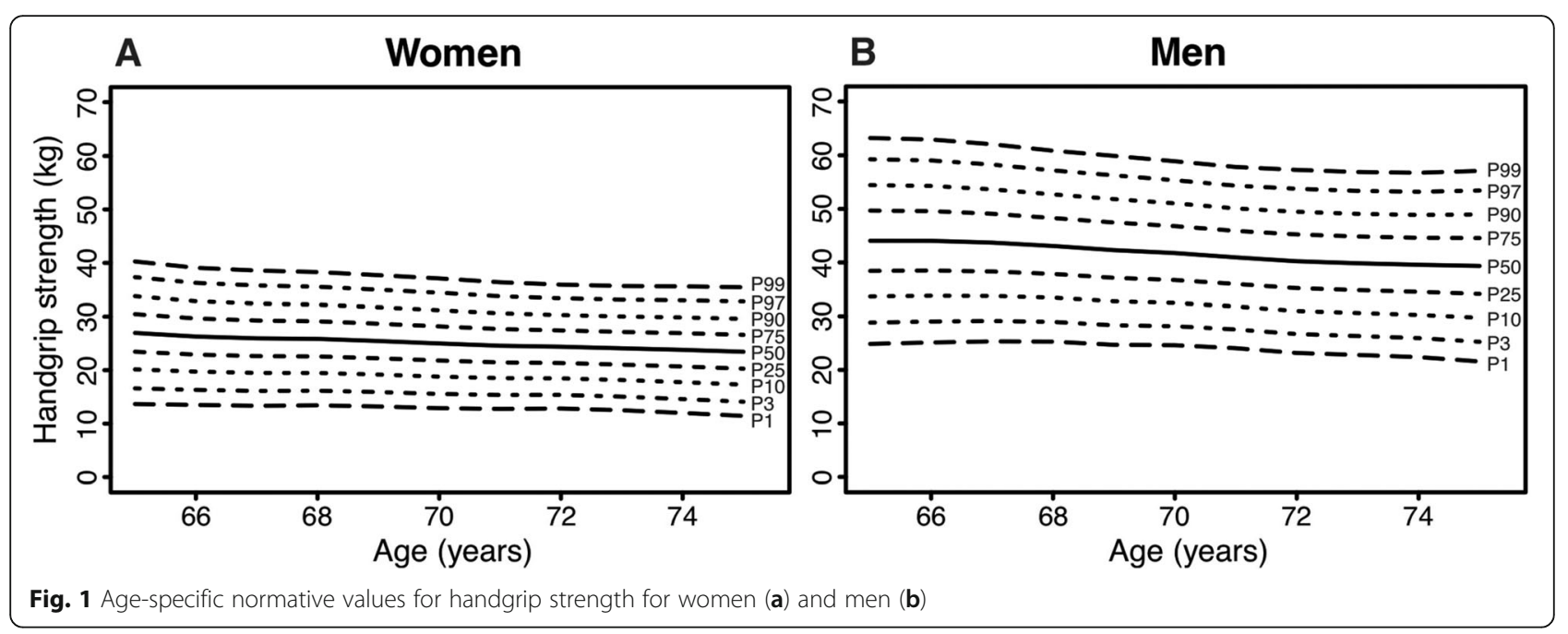



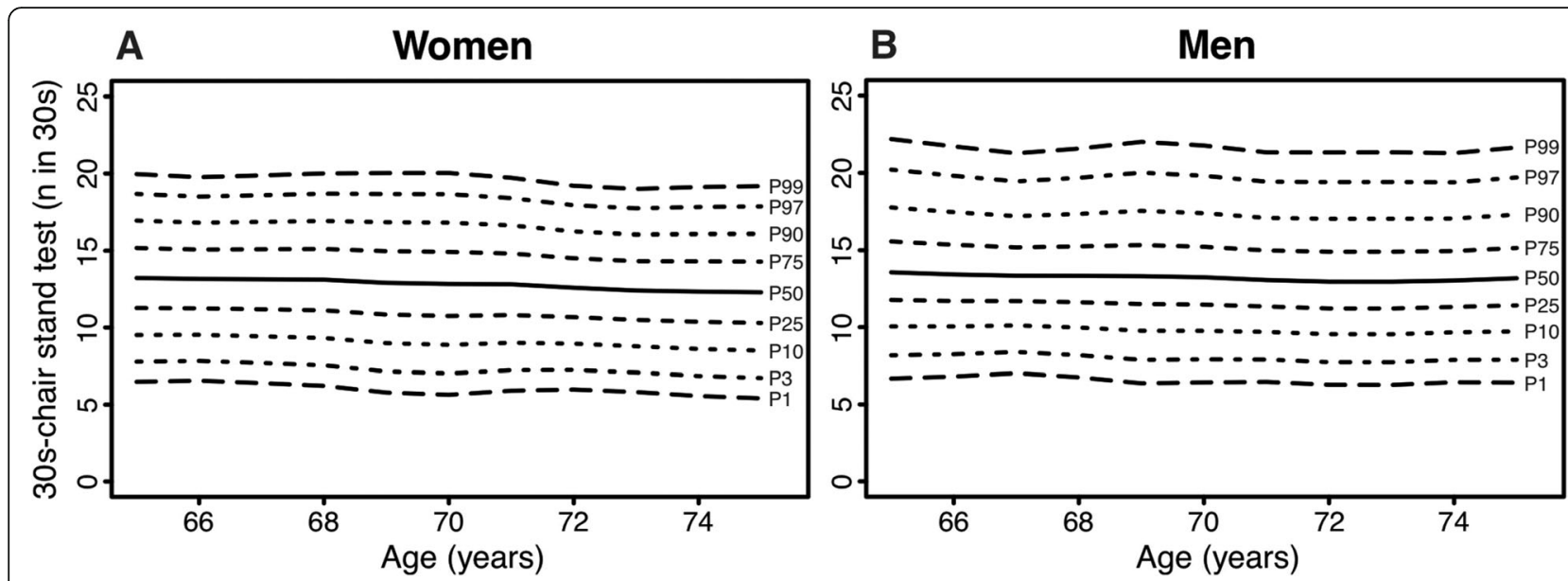

Fig. 2 Age-specific normative values for the 30s-chair stand test for women (a) and men (b)

The sex differences with men performing higher in endurance and muscle strength domains as well as the superiority of women in the flexibility domains are in line with other studies [9-14, 16, 28-31]. This also applies to the age-specific decline in test results [9-16, 28-31]. Yet, specific values differ between studies. The normative values for handgrip strength provided in this paper are overall higher than other published values [14, 2831]. Handgrip strength is positively correlated to body height $[28,32,33]$. This could be one reason for the differences as the participants in our study were considerably taller. In the 30s-chair stand test and the 2 min-step test, our normative values for both women and men are lower than most of the other values reported [9-11, 1316]. One possible explanation could be the differences in body weight. Only some studies reported the body weight of their participants, but in most studies that did, the participants weighted less than ours $[9,11,14,15]$. For the 2 min-step test, only Chung et al. reported lower values [14]. Chen et al. provided similar normative values for the 30s-chair stand test [10]. There is indication that flexibility is dependent on culture [34]. For example, older adults in Hong Kong like to engage in "light Chinese-style mind-body exercise" with a focus on flexibility [14]. The normative values for Hong Kong for both, the sit-and-reach test and the back scratch test, are higher than ours [14]. This is also the case for the normative values of the sit-and-reach test for an older Taiwanese population [10]. Older men from Poland reached higher values in both flexibility tests while the values from older Polish women are similar to ours [16]. Apart from older men in one Spanish study [12], normative values from Spain and Portugal are lower for both flexibility tests [11-13]. Chilean women reached similar values in the sit-and-reach test and lower values in the back scratch test [15]. For the USA, Rikli \& Jones reported a narrower range of values with higher values in the lower percentiles and lower values in the higher percentiles for the sit-and-reach test and overall higher values for the back scratch test [9].
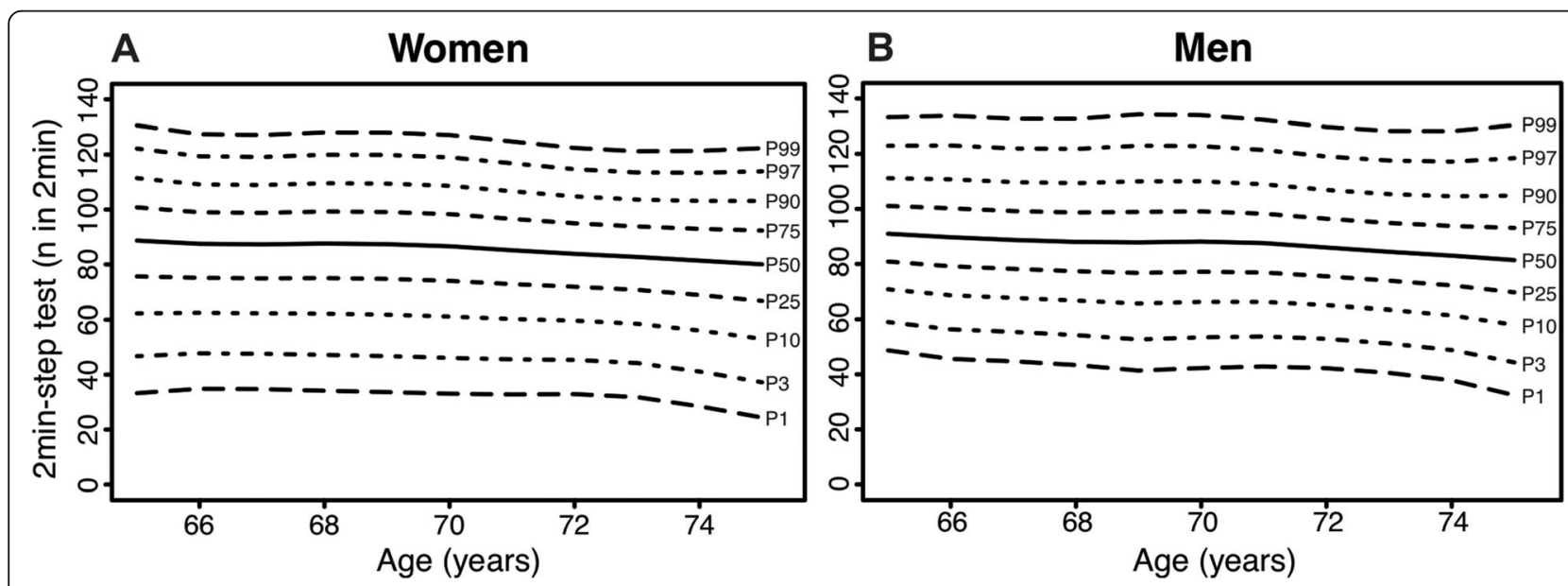

Fig. 3 Age-specific normative values for the 2 min-step test for women (a) and men (b) 

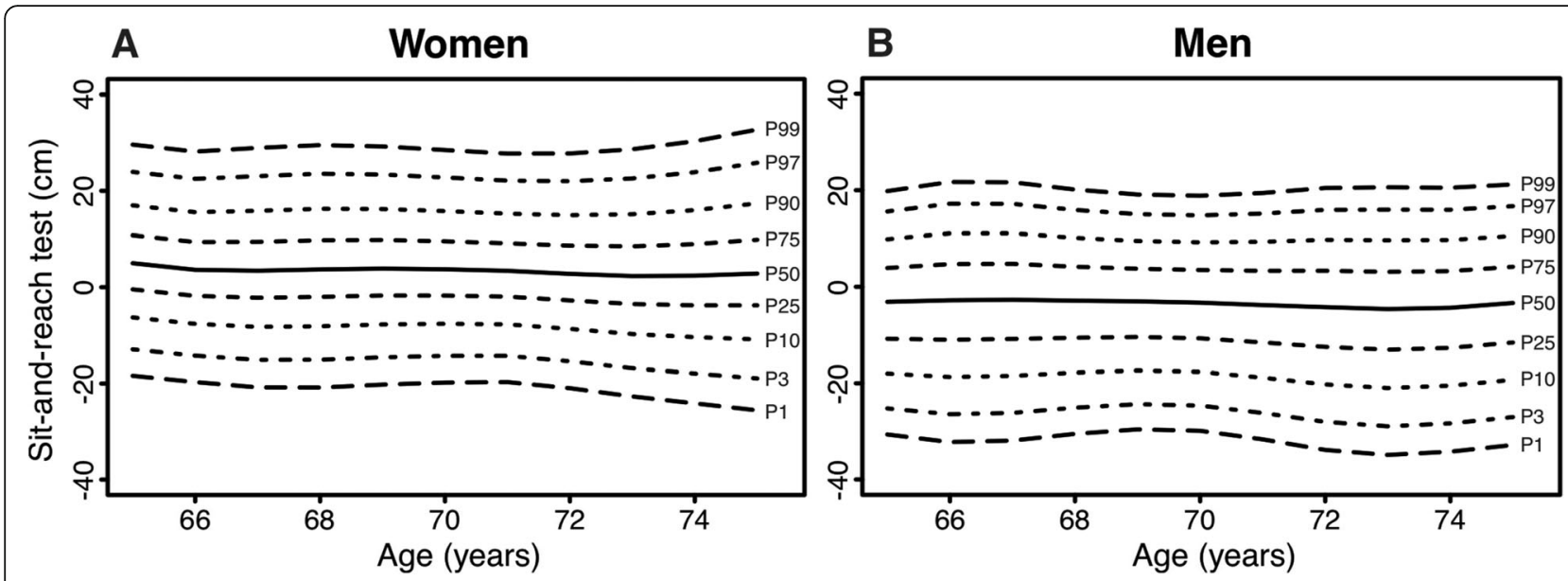

Fig. 4 Age-specific normative values for the sit-and-reach test for women (a) and men (b)

The representativeness of the study sample for the older German population must be considered with caution. In comparison to national census data of 60-69year-olds in 2011 [35] the study sample was better educated (22.0\% (census 2011) vs. 31.6\% (OUTDOOR ACTI VE) with Abitur (German equivalent to a high school diploma). Since there is a positive association between education and physical fitness [36], this could lead to an overestimation of normative values. All participants lived in Bremen, a city in the northwest of Germany. According to previously published studies, health-related behaviour e.g. physical activity differs between urban and rural areas $[37,38]$, however, there is yet no clear picture in which direction. Moreover, the included sub-districts in Bremen are highly heterogeneous. This is also reflected in the land use mix. Proportions of agricultural land use of the included sub-districts range from $0 \%$ (Neustadt and Ostertor) to $59.8 \%$ (Arbergen), thus a range of diverse areas is covered in the sample. Although disparities are slowly diminishing in the third decade after German reunification, prevalence of overweight and obesity still differs systematically between East and West Germany [39], and also body height is geographically patterned in Germany [40]. This might lead to overestimation of handgrip normative values and underestimation of chair stand normative values for Germany. In our study, we excluded institutionalized persons. In the age group 65 to 75 years, only a small proportion $(1.11 \%)$ is in residential care [41], therefore, the normative values are probably not seriously impacted by this limitation. The OUTDOOR ACTIVE participants were able to participate in the study without taking part in the physical fitness tests. Participants of the physical fitness test were less likely to report only medium or poor subjective health compared to the other 433 survey participants (14.9\% versus $26.3 \%$ ) and less likely to be under constant medication $(72.9 \%$ versus $76.7 \%$ ) leading to a probable overestimation of

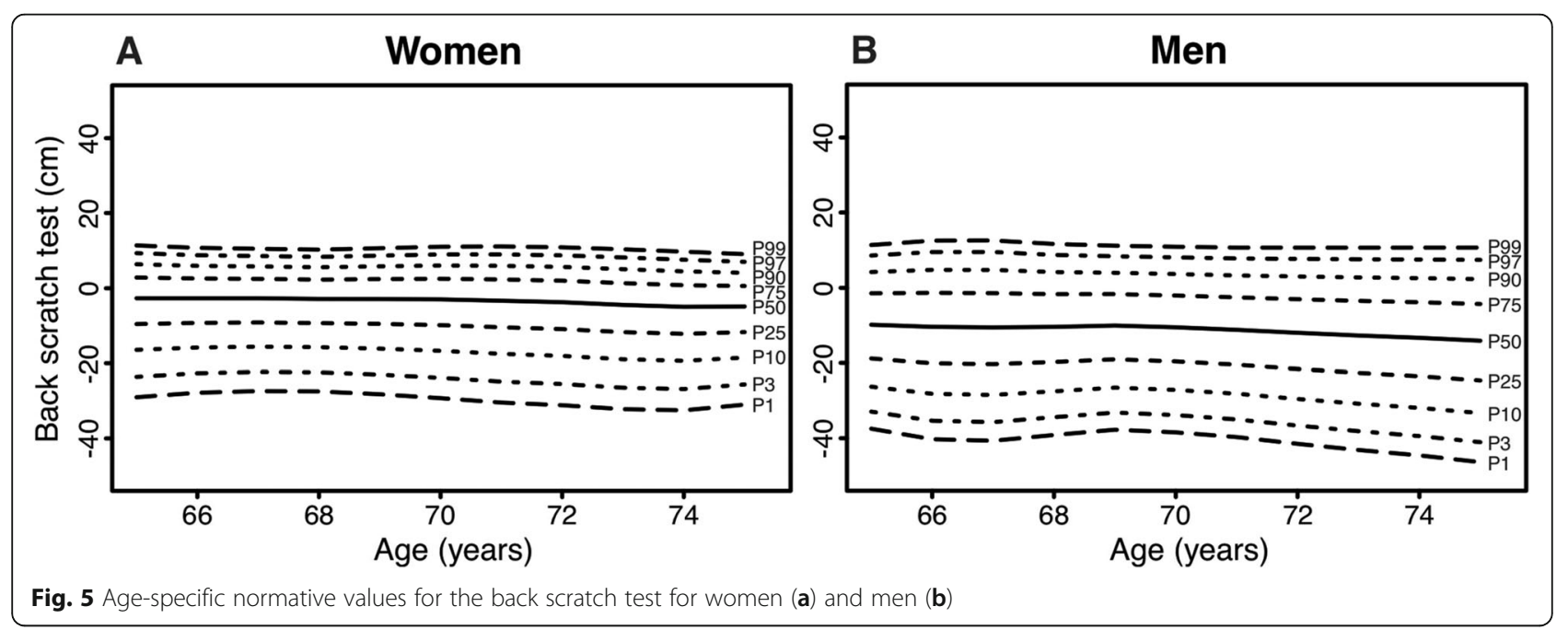


normative values. This is a well-known limitation also in other studies [42]. One particular strength of the study is the use of the GAMLSS method, which gives normative values for each year of life and not only age groups.

\section{Conclusions}

The present study is, to the best of our knowledge, the first to provide normative values for handgrip strength and components of the Senior Fitness Test in older adults aged 65 to 75 years residing in Germany. They might be useful in future research by providing evidence-based meaningful cut-offs for the investigated measures. Furthermore, they can be used for categorizing test results in (non-)clinical practice, and thus, supporting elaborated feedback to test participants.

\section{Abbreviations}

GAMLSS: Generalized additive models for location, scale, and shape; ISCE D: International Standard Classification of Education

\section{Supplementary Information}

The online version contains supplementary material available at https://doi. org/10.1186/s12877-021-02188-9.

Additional file 1. Response variable distributions of the included

models (selection based on the Akaike information criterion).

Additional file 2. Number of participants by sex and age.

Additional file 3. Means, standard deviations, and standard errors for all physical fitness measurements by sex and age.

Additional file 4. Tabulated sex- and age-specific normative values for handgrip strength $(\mathrm{kg})(\mathrm{A})$, the 30s-chair stand test ( $\mathrm{n}$ in 30s) (B), the 2 min-step test ( $\mathrm{n}$ in $2 \mathrm{~min}$ ) (C), the sit-and-reach test $(\mathrm{cm})(\mathrm{D})$, and the back scratch test $(\mathrm{cm})(\mathrm{E})$

\section{Acknowledgements}

The authors would like to thank all participants of the OUTDOOR ACTIVE study.

\section{Authors' contributions}

BMA performed statistical analyses. KB contributed to the conception and design of the study as well as statistical analyses. BMA and KB drafted the manuscript. IS critically revised and reviewed the manuscript. All authors read and approved the final manuscript.

\section{Funding}

The OUTDOOR ACTIVE project is funded by the German Federal Ministry of Education and Research, (BMBF; grant numbers 01EL1422B and 01EL1822B). The content of this article reflects only the authors' views and the funder is not liable for any use that may be made of the information contained therein. Open Access funding enabled and organized by Projekt DEAL.

\section{Availability of data and materials}

The datasets used and/or analysed during the current study are available from the corresponding author on reasonable request.

\section{Declarations}

Ethics approval and consent to participate

The OUTDOOR ACTIVE study was approved by the ethical committee of the University of Bremen. All participants provided written informed consent.

Consent for publication

Not applicable.

\section{Competing interests}

The authors declare that they have no competing interests.

Received: 14 October 2020 Accepted: 2 April 2021

Published online: 26 April 2021

\section{References}

1. Sardinha LB, Cyrino ES, Santos LD, Ekelund U, Santos DA. Fitness but not weight status is associated with projected physical independence in older adults. Age (Dordr). 2016;38(3):54. https://doi.org/10.1007/s11357-0169911-4.

2. Pereira C, Baptista F, Cruz-Ferreira A. Role of physical activity, physical fitness, and chronic health conditions on the physical independence of community-dwelling older adults over a 5-year period. Arch Gerontol Geriatr. 2016;65:45-53. https://doi.org/10.1016/j.archger.2016.02.004.

3. Knapik A, Brzęk A, Famuła-Wąż A, Gallert-Kopyto W, Szydłak D, Marcisz C, et al. The relationship between physical fitness and health self-assessment in elderly. Medicine (Baltimore). 2019;98(25):e15984. https://doi.org/10.1097/ MD.0000000000015984.

4. Chung PK, Zhao Y, Liu JD, Quach B. A canonical correlation analysis on the relationship between functional fitness and health-related quality of life in older adults. Arch Gerontol Geriatr. 2017;68:44-8. https://doi.org/10.1016/j.a rchger.2016.08.007.

5. Garatachea N, Molinero O, Martínez-García R, Jiménez-Jiménez R, GonzálezGallego J, Márquez S. Feelings of well being in elderly people: relationship to physical activity and physical function. Arch Gerontol Geriatr. 2009;48(3): 306-12. https://doi.org/10.1016/j.archger.2008.02.010.

6. Milanović Z, Pantelić S, Trajković N, Sporiš G, Kostić R, James N. Age-related decrease in physical activity and functional fitness among elderly men and women. Clin Interv Aging. 2013;8:549-56. https://doi.org/10.2147/CIA S44112.

7. Lee YS, Chang LY, Chung WH, Lin TC, Shiang TY. Does functional fitness decline in accordance with our expectation? - a pilot study in healthy female. BMC Sports Sci Med Rehabil. 2015;7(1):17. https://doi.org/10.1186/ s13102-015-0012-y.

8. Rikli RE, Jones CJ. Development and validation of a functional fitness test for community-residing older adults. J Aging Phys Act. 1999;7(2):129-61. https://doi.org/10.1123/japa.7.2.129.

9. Rikli RE, Jones CJ. Functional fitness normative scores for communityresiding older adults, ages 60-94. J Aging Phys Act. 1999;7(2):162-81. https://doi.org/10.1123/japa.7.2.162.

10. Chen HT, Lin CH, Yu LH. Normative physical fitness scores for communitydwelling older adults. J Nurs Res. 2009;17(1):30-41. https://doi.org/10.1097/ JNR.0b013e3181999d4c

11. Pedrero-Chamizo R, Gómez-Cabello A, Delgado S, Rodríguez-Llarena S, Rodríguez-Marroyo JA, Cabanillas E, et al. Physical fitness levels among independent non-institutionalized Spanish elderly: the elderly EXERNET multi-center study. Arch Gerontol Geriatr. 2012;55(2):406-16. https://doi. org/10.1016/j.archger.2012.02.004

12. Gusi N, Prieto J, Olivares PR, Delgado S, Quesada F, Cebrián C. Normative fitness performance scores of community-dwelling older adults in Spain. J Aging Phys Act. 2012;20(1):106-26. https://doi.org/10.1123/japa.20.1.106.

13. Marques EA, Baptista F, Santos R, Vale S, Santos DA, Silva AM, et al. Normative functional fitness standards and trends of Portuquese older adults: cross-cultural comparisons. J Aging Phys Act. 2014;22(1):126-37. https://doi.org/10.1123/japa.2012-0203.

14. Chung PK, Zhao Y, Liu JD, Quach B. Functional fitness norms for community-dwelling older adults in Hong Kong. Arch Gerontol Geriatr. 2016;65:54-62. https://doi.org/10.1016/j.archger.2016.03.006

15. Valdés-Badilla P, Concha-Cisternas Y, Guzmán-Muñoz E, Ortega-Spuler J, Vargas-Vitoria R. Reference values for the senior fitness test in Chilean older women. Rev Med Chil. 2018;146(10):1143-50. https://doi.org/10.4067/S003498872018001001143

16. Ignasiak Z, Sebastjan A, Slawinska T, Skrzek A, Czarny W, Krol P, et al. Functional fitness normative values for elderly polish population. BMC Geriatr. 2020;20(1):384. https://doi.org/10.1186/s12877-020-01787-2.

17. Forberger S, Bammann K, Bauer J, Boll S, Bolte G, Brand T, et al. How to tackle key challenges in the promotion of physical activity among older adults (65+): The AEQUIPA network approach. Int J Environ Res Public Health. 2017;14(4):379. https://doi.org/10.3390/ijerph14040379. 
18. Bammann K, Drell C, Lübs LL, Stalling I. Cluster-randomised trial on participatory community-based outdoor physical activity promotion programs in adults aged 65-75 years in Germany: protocol of the OUTDOOR ACTIVE intervention trial. BMC Public Health. 2018;18(1):1197. https://doi.org/10.1186/s12889-018-6124-z.

19. Rikli RE, Jones CJ. Senior Fitness Test manual. 2 ed: Human Kinetics; 2013.

20. The 4-Stage Balance Test: Centers for Disease Control and Prevention. https://www.cdc.gov/steadi/pdf/STEADI-Assessment-4Stage-508.pdf. Accessed 14 Feb 2021

21. Mackenbach J, Avendano M, Andersen-Ranberg K, Aro AR. 3.1 Physical health. In: Börsch-Supan A, Brugiavini A, Jürges H, Mackenbach J, Siegrist J, Weber G, editors. Health, ageing and retirement in Europe. First results from the Survey of Health, Ageing and Retirement in Europe. Mannheim: Mannheim Research Institute for the Economics of Aging (MEA); 2005.

22. Obesity: preventing and managing the gobal epidemic. WHO; 2000

23. International Standard Classification of Education. ISCED 1997. Paris: UNESCO; 2006.

24. Jenkinson C, Wright L, Coulter A. Criterion validity and reliability of the SF36 in a population sample. Qual Life Res. 1994;3(1):7-12. https://doi.org/10.1 007/BF00647843.

25. Cole TJ. The LMS method for constructing normalized growth standards. Eur J Clin Nutr. 1990:44(1):45-60.

26. Rigby RA, Stasinopoulos DM. Generalized additive models for location, scale and shape. J Royal Stat Soc. 2005;54(3):507-54. https://doi.org/10.1111/j.14 67-9876.2005.00510.x.

27. Stasinopoulos DM, Rigby RA. Generalized additive models for location scale and shape (GAMLSS) in R. J Stat Softw. 2007;23(7):46.

28. Mendes J, Amaral TF, Borges N, Santos A, Padrão P, Moreira P, et al. Handgrip strength values of Portuguese older adults: a population based study. BMC Geriatr. 2017;17(1):191. https://doi.org/10.1186/s12877-017-0590-5.

29. Turusheva A, Frolova E, Degryse JM. Age-related normative values for handgrip strength and grip strength's usefulness as a predictor of mortality and both cognitive and physical decline in older adults in Northwest Russia. J Musculoskelet Neuronal Interact. 2017;17(1):417-32.

30. Alqahtani B, Alenazi A, Alshehri M, Alqahtani M, Elnaggar R. Reference values and associated factors of hand grip strength in elderly Saudi population: a cross-sectional study. BMC Geriatr. 2019;19(1):271. https://doi. org/10.1186/s12877-019-1288-7.

31. Ramírez-Vélez R, Correa-Bautista JE, García-Hermoso A, Cano CA, Izquierdo M. Reference values for handgrip strength and their association with intrinsic capacity domains among older adults. J Cachexia Sarcopenia Muscle. 2019;10(2):278-86. https://doi.org/10.1002/jcsm.12373.

32. Wearing J, Konings $P$, Stokes $M$, de Bruin ED. Handgrip strength in old and oldest old Swiss adults - a cross-sectional study. BMC Geriatr. 2018;18(1): 266. https://doi.org/10.1186/s12877-018-0959-0.

33. Maranhao Neto GA, Oliveira AJ, Pedreiro RC, Pereira-Junior PP, Machado S, Marques Neto $S$, et al. Normalizing handgrip strength in older adults: an allometric approach. Arch Gerontol Geriatr. 2017;70:230-4. https://doi.org/1 0.1016/j.archger.2017.02.007.

34. Nguyen HM, Cihlar V. Differences in physical fitness and subjectively rated physical health in Vietnamese and German older adults. J Cross Cult Gerontol. 2013;28(2):181-94. https://doi.org/10.1007/s10823-013-9195-4.

35. Personen: Höchster Schulabschluss - Alter (5er-Jahresgruppen)/ Erwerbsstatus (ausführlich)/Höchster beruflicher Abschluss/Religion. Statistische Ämter des Bundes und der Länder. 2011. https://ergebnisse. zensus2011.de/datenbank//online?operation=table\&code=2000S-2037\&bypa ss=true\&levelindex=0\&levelid $=1613554730491$ \#abreadcrumb. Accessed 16 Feb 2021.

36. Valkeinen H, Harald K, Borodulin K, Mäkinen TE, Heliövaara M, Leino-Arjas P, et al. Educational differences in estimated and measured physical fitness. Eur J Pub Health. 2013;23(6):998-1002. https://doi.org/10.1093/eurpub/ckt049.

37. Dyck DV, Cardon G, Deforche B, De Bourdeaudhuij I. Urban-rural differences in physical activity in Belgian adults and the importance of psychosocial factors. J Urban Health. 2011;88(1):154-67. https://doi.org/10.1007/s11524-01 0-9536-3.

38. Drygas W, Kwaśniewska M, Kaleta D, Pikala M, Bielecki W, Głuszek J, et al. Epidemiology of physical inactivity in Poland: prevalence and determinants in a former communist country in socioeconomic transition. Public Health. 2009;123(9):592-7. https://doi.org/10.1016/j.puhe.2009.08.004.
39. Prütz F, Rommel A, Kroll LE, Lampert T. 25 Jahre nach dem Fall der Mauer Regionale Unterschiede in der Gesundheit. GBE kompakt. 2014;5(3):1-14. https://doi.org/10.25646/3054.

40. Hiermeyer M. Height and BMI values of German conscripts in 2000, 2001 and 1906. Econ Hum Biol. 2009;7(3):366-75. https://doi.org/10.1016/j.ehb.2 009.07.005.

41. Pflegebedürftige in Pflegeheimen (Anzahl). Gliederungsmerkmale: Jahre, Deutschland, Alter, Träger, Pflegegrad: GBE Bund. 2017. http://www.gbebund.de/oowa921-install/servlet/oowa/aw92/dboowasys921.xwdevkit/xwd_ init?gbe.isgbetol/xs_start_neu/\&p_aid=i\&p_aid=12860590\&nummer $=888 \&$ _ sprache=D\&p_indsp=-\&p_aid=11572312. Accessed 05 Oct 2020

42. Shrank WH, Patrick AR, Brookhart MA. Healthy user and related biases in observational studies of preventive interventions: a primer for physicians. J Gen Intern Med. 2011;26(5):546-50. https://doi.org/10.1007/s11606-010-1 609-1.

\section{Publisher's Note}

Springer Nature remains neutral with regard to jurisdictional claims in published maps and institutional affiliations.
Ready to submit your research? Choose BMC and benefit from:

- fast, convenient online submission

- thorough peer review by experienced researchers in your field

- rapid publication on acceptance

- support for research data, including large and complex data types

- gold Open Access which fosters wider collaboration and increased citations

- maximum visibility for your research: over $100 \mathrm{M}$ website views per year

At BMC, research is always in progress.

Learn more biomedcentral.com/submissions 